\title{
Adhesivos para prōtesis completas: situación actual
}

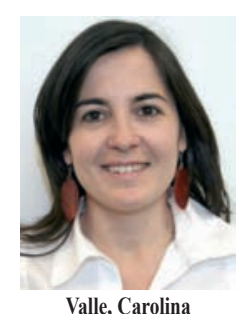

\author{
Adhesives for complete dentures: present situation
}

Valle Rodríguez, Carolina*
Godou Rico, Laura*
García Evans, Ofelia*
Pradíes Ramirí, Guillermo **
* Máster en Prótesis Bucofacial.
** Profesor Titular.
Departamento de Estomatología I.
Facultad de Odontología u.C.M.

\section{Correspondencia}

Guillermo Pradíes Ramiro

Facultad de Odontología

Universidad Complutense de Madrid

Plaza de Ramón y Cajal s/n

28040 Madrid, España

Email: gpradies@odon.ucm.es
Resumen: Los adhesivos para dentaduras son sustancias utilizadas para aportar una mayor retención a las prótesis. Su uso se remonta al siglo XVIII, pero en la literatura dental no se mencionó hasta el XIX. En la actualidad son fabricados por casas farmacéuticas, y proporcionan viscosidad y pegajosidad cuando absorben agua. Se comercializan en diferentes formas: polvo, cremas o pastas, tiras y almohadillas adhesivas.

A pesar de su posible extendida utilización dentro de la población con prótesis completas, muchos dentistas han mantenido a lo largo de la historia una actitud reticente y escéptica sobre su indicación, utilización y efectividad. En otros casos, la utilización de adhesivos se ha considerado dentro del campo de los prostodoncistas como un fracaso en la técnica de realización de la prótesis completa, entendiendo que si era necesaria la utilización del mismo era porque la prótesis no estaba "bien hecha». Sin embargo, los laboratorios farmacéuticos han continuado su investigación, desarrollo y promoción, y la demanda por parte del paciente, sigue aumentando, aún a pesar de la creciente aplicación de las técnicas implantológicas.

Debido a esta paradoja existente, se decidió realizar un análisis de revisión de las principales ventajas o inconvenientes que determinarán su uso y en qué casos se deberán indicar y cuándo no. Además, se efectuó una encuesta en farmacias de distintas comunidades autónomas, que refleja qué adhesivos existen actualmente en España, cuál es su precio y su forma de presentación así como, cuáles se utilizan más y qué opinión tienen los pacientes sobre los mismos.

Palabras clave: Adhesivos. Prótesis Completa. Retención. Estabilidad,

Abstract: The use of adhesives to improve the hold of complete dentures goes back to the $18^{\text {th }}$ century. Since then, the pharmaceutical industry has introduced different compounds with the aim of providing viscosity and stickiness when absorb water, and in this way improving the retentiveness of removable prosthesis. These adhesives have been composed of rubber, pectins, methylcellulose, hydroxyl-methylcellulose, carboxyl-methylcellulose, sodium-cellulose and synthetic polymers that improve denture support, together with other compounds, such as antimicrobial agents, additives, colourings and preservatives. They have been marketed in different vehicles such as: powder, paste, cream and adhesive cushions.

In spite of its wide use among complete denture bearers, dentists have usually maintained a negative attitude on the use and indications of denture adhesives. There is currently a controversy between those who defend its use as a mean to increase complete denture retention and others who still believe that a well done complete denture doesn't require any kind of adhesive, since it should hold by itself.

In spite of these controversies the number of adhesive products on the market is increasing and the number of potential users is also on the rise, what makes research in this area of high importance.

Key words: Adhesive materials. Complete denture. Retention. Stability

BIBLID [1138-123X (2007)12:4; octubre-diciembre 209-316]

Valle Rodríguez C, Godoy Rico L, García Evans O, Pradíes Ramiro G. Adhesivos para prótesis completas: situación actual. RCOE 2007;12(4):273-282. 


\section{Introducción}

El progreso de la Odontología en los últimos años es un hecho innegable, y desde la aparición de los implantes dentales osteointegrados, las posibilidades de tratamiento se han multiplicado. Con respecto a los desdentados totales con o sin reabsorciones extremas de los rebordes residuales, la posible falta de estabilidad de las prótesis convencionales, o la incapacidad psicológica para llevarlas, la implantoprótesis se considera hoy en día la mejor solución para rehabilitarlos.

Pero aún así, existen situaciones, como en el caso de los pacientes gravemente inmunodeprimidos, patología tumoral terminal, reabsorciones extremas del hueso residual, o simplemente factores económicos, en los que la prótesis completa convencional sigue siendo la alternativa para su tratamiento.

En ocasiones, los problemas biomecánicos de retención, soporte y estabilidad, se encuentran principalmente en un diagnóstico impreciso, en un discutible diseño y en una ejecución del tratamiento insuficiente por parte del odontólogo. En estos casos, una nueva realización de la prótesis según la teoría y técnica adecuada, puede solucionar el problema. Pero existen casos en los que a pesar de realizar un tratamiento protésico correcto, las características del sustrato sobre las que van colocadas, no permite un adecuado comportamiento biomecánico, o al menos, no el que el paciente desea. En estos casos, el paciente recurre en numerosas ocasiones, con o sin la prescripción facultativa a la utilización de mecanismos alternativos de retención, como son los adhesivos para prótesis.

\section{Adhesivos para prótesis: antecedentes y conside- raciones generales}

Las primeras referencias bibliográficas de los adhesivos aparecen en el siglo XIX, pero su uso se remonta al siglo XVIII. La primera patente apareció en Estados Unidos en 1913 y ya en 1939 se calculaba que más de quince millones de portadores de prótesis los utilizaban, existiendo múltiples empresas dedicadas a su fabricación ${ }^{1-2}$.

A pesar de este gran auge, tanto en la demanda por parte de los pacientes como en la oferta por parte de las empresas farmacéuticas, los dentistas mantenían una actitud negativa a su utilización, y en 1945 apareció un artículo donde se enumeraban las posibles razones por las que se podría utilizar los adhesivos, siendo una de ellas "cuando el dentista es incompetente e incapaz de realizar una prótesis correcta y ajustada a la boca de los pacientes»".

En 1991, Shay ${ }^{2-3}$ describió el mecanismo por el cual el adhesivo, una vez colocado en la boca, es capaz de pro- ducir fuerzas retentivas que mantengan la prótesis en su lugar. Según este autor el volumen del adhesivo cambia al mezclarse con el agua, de un 50\% a un $150 \%$, consiguiendo así rellenar el espacio existente entre la prótesis y Ios tejidos. El adhesivo absorbe el agua gracias a la atracción que se produce entre sus aniones y los cationes de la mucosa proteica tisular. Como la saliva aumenta la viscosidad del adhesivo, se aumenta la fuerza que se requiere para separar la prótesis de los tejidos $^{1-3}$. La tabla 1 muestra las propiedades ideales que según la ADA, deben cumplir los adhesivos para prótesis completas extraíbles. De igual manera, la tabla 2 muestra los componentes químicos básicos de los pegamentos para dentaduras 4 .

En opinión de algunos autores, el uso de los adhesivos da a los pacientes seguridad, tanto física como psicológica frente a la desinserción de sus prótesis, favorece la masticación, y gracias al efecto de relleno que proporcionan, disminuyen el acúmulo de partículas bajo las prótesis. Por otra parte, disminuyen el movimiento de las prótesis aportando mayor comodidad y confianza. Sin embargo, en el otro lado de la balanza los pacientes

\begin{tabular}{|c|c|}
\hline - Ausencia de toxicidad & $\begin{array}{l}\text { - Buenas propiedades organolépticas: } \\
\text { sabor, color, olor, etc. }\end{array}$ \\
\hline - Biocompatibilidad con la mucosa & - No afectar al sentido del gusto \\
\hline $\begin{array}{l}\text { - Capacidad de inhibición del } \\
\text { crecimiento de microorganismos } \\
\text { adicional a la prótesis }\end{array}$ & - Proporcionar retención y estabilidad \\
\hline $\begin{array}{l}\text { - Preservación de la integridad } \\
\text { de la prótesis }\end{array}$ & - Bajo coste \\
\hline
\end{tabular}




\section{Tabla 2. Composición básica de los adhesivos para prótesesis completąas}

Grupo 1: materiales responsables de la adhesión: gomas, pectina, metilcelulosa, hidroximetilcelulosa, carboximetilcelulosa, celulosa sódica y polímeros sintéticos (acrílicos, polivinil acético, óxido polietileno, etc.)

Grupo 2: agentes antimicrobianos: tetraborato sódico, etanol, etc. Grupo 3: aditivos, conservantes y colorantes.

\section{Tabla 3. Instrucciones generales en el uso de adhesivos}

1. Limpiar e hidratar la superficie de la prótesis: eliminar, si existiese cualquier resto de adhesivo con algodón y disolvente de alcohol

2. Limpiar la mucosa oral de restos de pegamento anterior.

3. Aplicar pequeñas cantidades en la superficie de la prótesis:

- en la región de anterior, en el centro del paladar y en posterior para la prótesis superior

- en el surco del reborde alveolar extendiéndose desde anterior a distal

- mojar antes la prótesis si se va a aplicar en polvo

4. Colocar y presionar la prótesis durante 30-60 segundos:

- eliminar excesos

- instruir a los pacientes para que cierren en "céntrica» varias veces para que se distribuya el adhesivo quedando una fina capa sobre la mucosa.

han descrito sensaciones desagradables con respecto a la textura, olor y sabor. También con algunos tipos de adhesivos, los pacientes han encontrado dificultades a la hora de su aplicación.

Uno de los aspectos que está en discusión actualmente, es la posibilidad de que su uso prolongado pueda modificar la flora oral favoreciendo el crecimiento de Candida albicans, provocando candidiasis a los portadores de las prótesis. En este sentido, existen investigadores que defienden que los adhesivos, no solo no favorecen el crecimiento de Candida, si no que contienen en su composición agentes antimicrobianos que van a inhibir el crecimiento de este hongo. Mientras que otros, cuestionan o incluso apoyan tesis contrarias a estas como Bart et al en 1940 o Stafford y Rusell en 1971, encontraron que los adhesivos podrían servir como reservorio para el crecimiento de Candida albicans.

En cuanto a las formas de presentación de los mismos, se comercializan en forma de pasta o crema, polvo y almohadillas adhesivas.

Los criterios de selección en cuanto a la indicación concreta de una u otra forma de presentación, parece basarse más en preferencias personales del paciente que en criterios especialmente científicos, si bien es verdad que en general los pacientes suelen probar varias formas de presentación antes de decidirse por una determinada.

En relación con las instrucciones de uso, aunque existen pequeñas diferencias en función de la marca o la presentación utilizada, en general se deberían observar las normas descritas en la tabla 3.

\section{Ventajas, incovenientes, indicaciones 4 contraindicaciones}

Un gran número de estudios evidencia que el uso de adhesivos no sólo consigue disminuir el movimiento de las prótesis, sino que también consigue mejorar la función masticatoria ya que elevan el coeficiente de la interfase, impidiendo así que la prótesis se separe de los tejidos de soporte ${ }^{5-9}$.

Dichos estudios «in vivo» han utilizado básicamente magnetómetros y registros cinetográficos para el registro de las variables estudiadas, demostrando sobre todo, que las prótesis mandibulares mejoran su comportamiento.

Debido a un uso continuado de las prótesis completas convencionales, se generan puntos de presión sobre los procesos alveolares que favorecen la reabsorción ósea y que tienen como consecuencia una mayor movilidad de las prótesis.

Como Shay ${ }^{3}$ demostró, el adhesivo al mezclarse con el agua cambia su volumen de un $50 \%$ a un $150 \%$, rellenando así el desajuste existente entre prótesis y tejidos. Así pues, el efecto de relleno de los adhesivos en prótesis que se encuentran desadaptadas 
frente al sustrato con el que se relaciona es una de las indicaciones más frecuentes, en espera de la realización de las nuevas prótesis.

Gracias también a este efecto de relleno, los adhesivos disminuyen el acumulo de partículas bajo las prótesis, reduciendo el mal olor y mal sabor y la posible irritación de la mucosa debida a el acúmulo de alimentos bajo las prótesis $5^{3,7-10}$. Sin embargo, otros autores opinan que el uso de adhesivos fomenta la existencia de partículas residuales que provocarían irritación y mal sabor a los pacientes.

Tarbet $^{10}$ afirma que aquellos pacientes en los que el uso continuado de las prótesis completas ha producido daño sobre el soporte periodontal (óseo y mucoso), pueden verse beneficiados del uso de adhesivos, ya que con ellos se disminuye el movimiento de las prótesis debido a los siguientes factores:

- la presión que la prótesis ejerce con el adhesivo sobre el soporte sería menor a la provocada directamente sobre los tejidos.

- ayuda a la distribución de fuerzas, disminuyendo la presión localizada sobre diferentes puntos.

- se reduce el trauma que se produce sobre los tejidos y la irritación sobre la mucosa, disminuyendo así la sensibilidad de los tejidos. Este extremo es también confirmado por otros autores como Grasso'.

De todos los posibles ${ }^{11-13}$ efectos atribuidos a lo adhesivos, el único que no genera ningún tipo de controversia entre los profesionales es el factor psicológico de seguridad que lleva a los portadores de prótesis a utilizarlos incluso cuando no los necesitan funcionalmente ${ }^{2}$.
Todas estas ventajas ${ }^{10-14}$ hacen que los adhesivos se consideren una opción terapéutica efectiva en los pacientes portadores de prótesis completas, siempre que los dentistas les den instrucciones para un buen uso de los mismos. Las indicaciones, de acuerdo a las ventajas que aporta su uso, son:

- Pacientes con hipersensibilidad en la mucosa, ya que se ve reducida con el uso de los adhesivos al conseguirse una mejor distribución de las fuerzas.

- Pacientes con rebordes muy reabsorbidos en los que la realización de una nueva prótesis no solucione el problema de movilidad, ya que con el uso de adhesivos se verá reducida.

- Pacientes con disminución de la autoestima, por su positivo efecto psicológico.

- Pacientes con hiposialia por la mejora de la interfase sustrato-prótesis (efecto de relleno).

- Retención de prótesis antiguas hasta la fabricación de una nueva.

Además de estas indicaciones que estarían en relación directa con el paciente, existen a nuestro juicio otras dos, relacionadas con nuestra propia actividad y que resultan de una indiscutible utilidad: en primer lugar la utilización de adhesivos para facilitar todos los registros de planchas base ${ }^{15}$, durante la elaboración de nuevas prótesis en pacientes con rebordes residuales muy reabsorbidos o de baja retención. En segundo lugar, cuando es necesario realizar impresiones de arrastre para la realización de arreglos parciales de dentaduras que impliquen pérdida del número de retenedores y que por lo tanto, no tengan una buena sujeción por sí mismas en la boca del paciente.
Por otro lado, se han descrito también efectos adversos relacionados con el uso de los adhesivos que muchas veces llevan a la incapacidad de los pacientes a poder utilizarlos.

En cuanto a los posibles inconvenientes del uso de adhesivos, no cabe duda de que su uso, sobre todo en manos poco hábiles o descuidadas, podría favorecer, por su efecto adhesivo, el acúmulo de placa. Este acúmulo de placa bacteriana se ha asociado en ocasiones a la inflamación y estomatitis encontrada en los tejidos de soporte de las prótesis ${ }^{15-18}$.

Es el dentista el que debe dar instrucciones adecuadas al paciente para mantener una higiene correcta y evitar así la irritación tisular. Además, las casas comerciales, en vista de este problema, están creando adhesivos menos viscosos que proporcionen la retención necesaria a las prótesis, pero a la vez facilite la limpieza de las mismas $^{19}$.

Otro inconveniente atribuido a los adhesivos es la provocación de náuseas y tos. Sin embargo, finalmente este fenómeno parece más relacionado con el inicial rechazo psicológico por parte de algunos pacientes al introducir una sustancia nueva en la boca.

En 1971 G. D. Stafford 20 , y después en 1994 J. E. Grasso', describieron que, en los primeros momentos de la aplicación de los adhesivos, los pacientes pueden tener una sensación de presión o como si las prótesis les apretara, lo cual dificultaría la retirada de las mismas. Estas sensaciones ocurren únicamente al inicio de aplicar el adhesivo y van disminuyendo poco a poco, según el paciente va adquiriendo habilidades para su manejo y desinserción. 
Se han descrito también problemas relacionados con la aplicación de los adhesivos y su dificultad de utilización. La mayoría de estos casos se han producido con los adhesivos en polvo, ya que requieren una mayor destreza manual para conseguir distribuir todo el polvo de manera adecuada por la superficie de la prótesis. Además, con este tipo de adhesivos, parte se pierde al introducirlo en la boca, lo que incomoda más a los pacientes.

Se han encontrado también alteraciones sistémicas asociadas al uso de adhesivos, como $2,9,10$ :

- Alteraciones gástricas, debido a la ingesta de los adhesivos (fundamentalmente con los polvos).

- Reacciones inflamatorias o enrojecimiento de las mucosas por sensibilidad a alguno de los componentes.

- Reacciones alérgicas a alguno de los componentes.

Conocidos los inconvenientes de los adhesivos, existen una serie de circunstancias en las que se considera una contraindicación utilizar los adhesivos:

- Situaciones en la que se ha demostrado la existencia de alergia al adhesivo $o$ a algunos de sus componentes.

- Pacientes con muy mala higiene, ya que el propio adhesivo va a dificultar la limpieza de la prótesis.

- Incapacidad psicológica para utilizar los adhesivos.

- Destreza manual limitada, que impida la colocación correcta y de manera homogénea del adhesivo por toda la prótesis.

- Prótesis rota y/o en muy mal estado, porque el adhesivo no puede garantizar no producir heridas debido al estado de la prótesis 0 , incluso, la ingesta accidental de fragmentos de la misma.

Efecto de los adhesivos sobre el crecimiento de Candida albicans.

Para que exista colonización por Candida albicans, es necesaria la adherencia del hongo a la superficie de los materiales de la prótesis $y, a$ partir de ahí, que se produzca el crecimiento y colonización.

Distintos autores ${ }^{20,21}$ han publicado que los adhesivos podrían servir como reservorio para el crecimiento de este hongo.

Para poder estudiar la relación Candida albicans $V s$ adhesivos para prótesis, lo que se estudia es la relación del pH medio con el número de colonias, ya que cuando el pH disminuye, aumenta el número de colonias. De acuerdo con esto, Stafford demostró que los adhesivos disminuyen el $\mathrm{pH}$ del medio (por debajo de 5,5 ) favoreciendo así el crecimiento fúngico ${ }^{22-24}$.

Desde 1997 autores como Nikawa y colaboradores ${ }^{22-27}$, han estudiado esta relación y han llegado a la conclusión de que lo que verdaderamente favorece que el hongo colonice y crezca, es el grado de higiene del paciente. Si los pacientes tienen una higiene adecuada, las probabilidades de que crezca este hongo son muy pequeñas.

Por otra parte Lo Muzio ${ }^{21}$ y colaboradores han recomendado la utilización de los adhesivos en aquellos pacientes con aftas orales o liquen plano erosivo porque contienen ingredientes como carboximetil celuIosa sódica, copolímeros de vinilo, etc. que ayudan a erradicar estas lesiones. Además han demostrado que los adhesivos liberan componentes (tetraborato sódico, hexoclorafeno, etc.) que inhiben el crecimiento de los hongos ${ }^{28-29}$.

Por tanto, parece que son los defectos en la higiene de las prótesis, y no los adhesivos, los que facilitan el crecimiento de microorganismos, por lo que es importante que los dentistas hagan un seguimiento exhaustivo de los pacientes y les enseñen las técnicas adecuadas de limpieza para sus prótesis.

\section{Formas de representación}

En cuanto a las formas de presentación de los adhesivos (pasta o crema, polvo, tiras o almohadillas adhesivas) todas son realizadas por casas farmacéuticas y tiene ingredientes comunes (carboximetil, celulosa, gomas vegetales, éter, etc.) que les proporcionan la viscosidad y pegajosidad necesaria para favorecer la adhesión. Pero cada una tiene unos componentes que les van a aportar propiedades diferenciadoras.

Los adhesivos en forma de polvo contienen óxido de magnesio, fosfato sódico y silicato cálcico que van a permitir crear un formato tipo polvo. Las pastas o cremas contienen aceites minerales de polietileno o vaselina que favorecen las propiedades de pegajosidad propias de las cremas. Las almohadillas adhesivas se componen de tres capas, las dos exteriores a base de celulosa pura en las que se intercalan fibras de acetato de celulosa. La capa interior está compuesta de dos gomas mezcladoras, alginato sódico y un monopolímero no iónico a base de polióxido de etileno ${ }^{1-3}$. 
En cuanto a la retención que proporcionan las distintas formas de presentación, Ghani y Picton (1994) ${ }^{16}$ realizaron un estudio para comprobar la retención que se consigue en las prótesis maxilares en función de la forma de presentación de los adhesivos, demostrando que la crema es la que menor resistencia ofrece a la desinsección y que los polvos son los que más fuerza pierden al cabo del tiempo. Estos resultados no coinciden con otros estudios ${ }^{829-31}$ según los cuales la crema era la que mayor retención proporcionaba.

Tarbet $^{10}$ ha estudiado el comportamiento de los polvos Vs. las cremas durante la función masticatoria. En sus estudios la crema era la que mayor retención proporcionaba, pero debido a su gran viscosidad, era también la que conseguía una peor adaptación de las prótesis. En cuanto a los adhesivos en polvo, otros autores demostraban que el polvo acababa eliminándose por la acción de la saliva en mayor medida que la cre$\mathrm{ma}^{30}$.

En cuanto a las preferencias de los pacientes, Coates publicó la opinión de diferentes pacientes recogida por medio de encuestas. En ellas se comprobó que los pacientes encontraban que la crema era el formato más sencillo de aplicar, el más duradero y con el que se encontraban más cómodo. Frente a las cremas, los adhesivos en polvo eran para los pacientes el formato de más difícil aplicación. Además, este estudio reflejaba que para los pacientes las almohadillas son las que mayor confort ofrecen para las prótesis mandibulares y para casos de rebordes muy reabsorbidos. Esto coincide con el estudio realizado por

\begin{tabular}{|c|c|c|c|c|}
\hline $\begin{array}{l}\text { Cuestionario } \\
\text { sobre el uso de adhesivos } \\
\text { para prótesis dentales } \\
\text { Marcar solo casillas afirmativas }\end{array}$ & \multicolumn{4}{|c|}{ Observaciones } \\
\hline $\begin{array}{l}\text { 1. Forma de adhesivo más de } \\
\square \quad \text { polvo }\end{array}$ & $\begin{array}{l}\text { emandada: } \\
\square \quad \text { almohac }\end{array}$ & dillas & $\square$ & crema \\
\hline $\begin{array}{l}\text { 2. Indicado por: } \\
\square \quad \text { paciente }\end{array}$ & $\square \quad$ farmacé & éutico & $\square$ & odontólogo \\
\hline $\begin{array}{l}\text { 3. Relación formato/precio } \\
\text { Polvo }\end{array}$ & Almohadillas & & Crema & \\
\hline 4. Casas comerciales: & $\begin{array}{l}\text { Polvo } \\
\text { Crema } \\
\text { Almohadillas }\end{array}$ & & & \\
\hline $\begin{array}{c}\text { 5. Publicidad: } \\
\square \quad \text { T.V. }\end{array}$ & farmacia & & & \\
\hline $\begin{array}{l}\text { 6. Quejas de los pacientes: } \\
\text { LIMPIEZA }\end{array}$ & SI $\square$ & NO $\square$ & PROT $\square$ & TEJ $\square$ \\
\hline MAL OLOR $\square$ & SI $\square$ & NO $\square$ & & \\
\hline MAL SABOR $\square$ & $\mathrm{SI} \square$ & NO $\square$ & & \\
\hline DIFICULTAD DE RETIRADA $\square$ & $\mathrm{SI} \square$ & NO $\square$ & & \\
\hline RETENCIÓN $\square$ & SI $\square$ & NO $\square$ & & \\
\hline
\end{tabular}

Figura 1. Cuestionario proporcionado a las farmacias.

Panagioutouni ${ }^{30}$ en 1995, donde se observó que la mayor retención la registraban las almohadillas.

En síntesis, según algunas de las publicaciones científicas revisadas ${ }^{19,28,30}$ se deduce que es preferible la utilización de crema a los polvos adhesivos. En el caso de las prótesis inferiores, cuando existe una gran reabsorción de los rebordes previa, o debida a las discrepancias que con el tiempo se producen entre las superficie de la prótesis y la progresión de la reabsorción de dicho reborde, las almohadillas por su efecto de relleno y amortiguación parecen ofrecer los mejores resultados.

\section{Encuesta}

Los datos mostrados a continuación corresponden con un cuestionario relacionado con distintos aspectos de la demanda de adhesivos para prótesis dentales en España. Se seleccionó aleatoriamente, una muestra de 40 farmacias pertenecientes a 3 comunidades autónomas distintas: Comunidad de Madrid, Castilla la Mancha y Comunitat Valenciana.

A todas las farmacias se les solicitó su colaboración como interlocutores entregándoles una memoria justificativa de los objetivos perseguidos así como las instrucciones para rellenar 


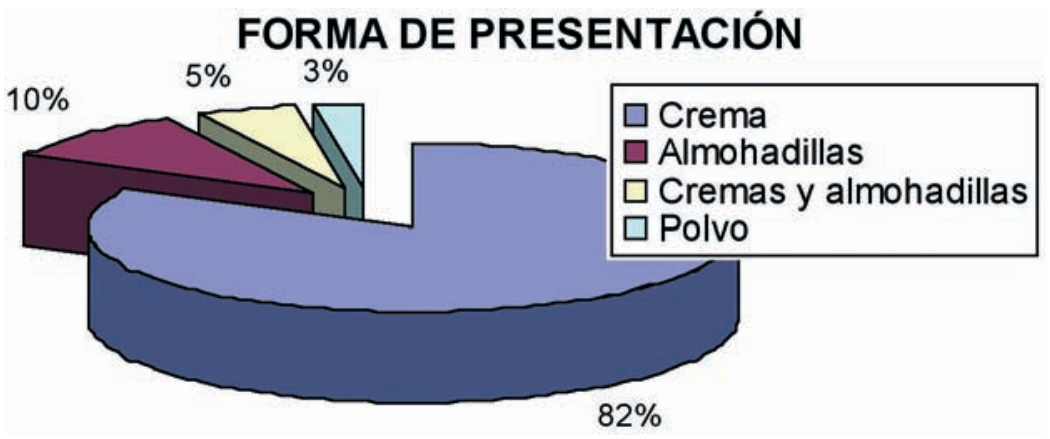

Figura 2. Diagrama sectorial de la demanda en función de la forma de presentación del adhesivo.

el cuestionario según el modelo adjunto (fig 1).

Los resultados que obtuvimos de las encuestas realizadas a las 40 farmacias, fueron los siguientes:

\section{Forma de presentación}

En cuanto a la forma de presentación, como podemos observar en la figura 2, la forma de presentación menos demandada son los polvos.

En el apartado de observaciones, muchas farmacias apuntaron que el polvo ni si quiera lo tenían, ya que era muy poco demandado y que los pacientes quedaban muy contentos con la crema.
Los resultados obtenidos se sometieron al test de la $X^{2}$ de Pearson que mostró resultados significativos a favor de la forma de presentación en crema ( $p$ d 0,0001).

\section{Prescripción}

Con esta pregunta pretendíamos saber si el paciente que demanda un determinado adhesivo es porque el odontólogo se lo había aconsejado (viene con receta), es el farmacéutico el que le recomienda un tipo u otro de adhesivo, o son los propios pacientes quienes compran el adhesivo sin que nadie se lo haya recomendado.
Las respuestas obtenidas quedan reflejadas en la gráfica de la figura 3.

Aunque podemos suponer un cierto sesgo en los resultados obtenidos en esta pregunta, ya que en muchas ocasiones el paciente pierde la receta o simplemente el odontólogo realiza una prescripción verbal, los resultados no ofrecen duda. De una forma mayoritaria y según el tést de la $X^{2}$, es el propio paciente el que se autoprescribe el adhesivo ( $p d 0,0001$ ).

\section{Relación formato/precio}

Se pretendía realizar un análisis genérico de la relación entre la forma de presentación y el precio, sin estar afectado por la marca comercial. Por esta razón, se entendió que la mejor forma de obtener resultados objetivos era preguntar a los propios farmacéuticos, quienes están acostumbrados a evaluar el precio de un producto asociado a otros factores como el número de aplicaciones posibles con cada forma o tamaño de aplicación, etc.

Los resultados obtenidos se muestran en la figura 4

En general, las farmacias coincidían en que aunque el precio de las cremas era mayor, finalmente el uso de las almohadillas resultaba más caro por-

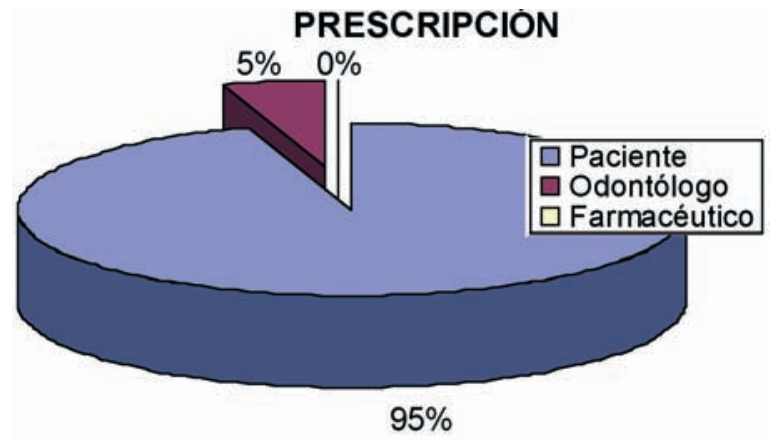

Figura 3. Distribución en función de la procedencia de la prescripción.

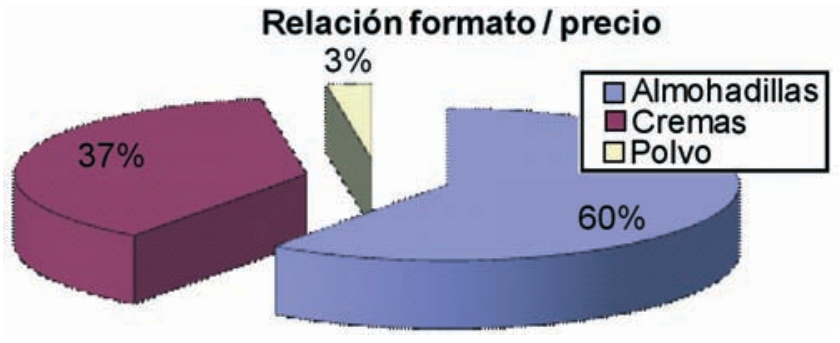

Figura 4. Gráfica de la relación formato / precio, que evidencia que la presentación en almohadillas es considerada como la más costosa, por parte de los farmacéuticos. 


\begin{tabular}{|c|c|c|c|}
\hline \multicolumn{4}{|c|}{ Tabla 4. Principales marcas de adhesivos para prótesis completa } \\
\hline Casas comerciales & Tipo & Tamaño/cantidad & Precio'06 \\
\hline COREGA ULTRA $^{\oplus}$ & polvo & mediano & $8,95 €$ \\
\hline COREGA ULTRA ${ }^{\circledast}$ & crema extra & mediano & $7,00 €$ \\
\hline FITTYDENT ${ }^{\circledR}$ & crema & mediano & $5,99 €$ \\
\hline FITTYDENT ${ }^{\oplus}$ & almohadilla & 6 unidades & $5,80 €$ \\
\hline NOVAFIX & crema & mediano & $7,45 €$ \\
\hline ALGASIV ${ }^{\circledR}$ & almohadilla & 6 unidades & $6,75 €$ \\
\hline PROTEFIX ${ }^{\circledast}$ & crema & mediano & $7,23 €$ \\
\hline PROTEFIX & polvo & mediano & $8,90 €$ \\
\hline STERADENT ${ }^{\oplus}$ & crema & mediano & $6,05 €$ \\
\hline STERADENT ${ }^{\oplus}$ & crema almohadilla & mediano & $6,65 €$ \\
\hline KUKIDENT PROV & crema & & \\
\hline
\end{tabular}

que con la crema hay para un mayor número de aplicaciones, mientras que en un paquete de almohadillas las aplicaciones están limitadas dependiendo del número de almohadillas de la caja (de 6 a 18).

Los resultados obtenidos se sometieron posteriormente al test de la $X^{2}$ de Pearson que mostró resultados significativos para la relación formato/precio de la crema ( $p$ d 0,0001).

\section{Casas comerciales}

En todas las farmacias encuestadas pudimos encontrar los mismos productos comerciales, la tabla 4 muestra una visión global de los principales aspectos de los mismos: precio, presentación, cantidad, etc.

\section{Publicidad}

En ninguna de las farmacias a las que se les realizó la encuesta se encontró publicidad de algún tipo de los adhesivos para prótesis (carteles, folletos informativos, etc.). Las farmacias señalaron que los representantes de adhesivos sólo dejan muestras, pero nunca dejan publicidad ni carteles. Sin embargo, la publicidad en televisión es, como todos conocemos, exhaustiva.

\section{Quejas de los pacientes}

En relación con esta variable, el farmacéutico preguntaba a sus clientes de confianza cual era su opinión sobre el adhesivo que utilizaban con respecto a las siguientes consideraciones:

\section{Limpieza}

Sólo 4 de las farmacias encuestadas reflejaron que los pacientes se quejaban de la dificultad de limpieza de las prótesis tras la aplicación del adhesivo.

\section{Mal olor}

Ninguna de las farmacias encuestada respondió que los pacientes se quejaran de mal olor con los adhesivos para prótesis.

\section{Mal sabor}

Las farmacias señalaron que antes los pacientes se quejaban de mal sabor con los adhesivos, sobre todo con las cremas. Pero en la actualidad, existen nuevas cremas que han intro- ducido entre sus componentes distintos productos como la manzanilla, capaces de mejorar sus propiedades organolépticas.

\section{Dificultad de retirada de la prótesis}

Ninguna de las farmacias reflejaron problemas por parte de los pacientes para retirar la prótesis tras la aplicación del adhesivo.

\section{Retención}

24 de las 40 farmacias contestó que a pesar de que los pacientes llevaran el adhesivo, se seguían quejando de que sus prótesis se movían y no se retenían. De esas 24 farmacias, 4 de ellas afirmaron que la forma de presentación en almohadillas era de la que más se quejaban los pacientes porque no se adaptaban bien a sus prótesis.

En el apartado de observaciones, algunas de las farmacias señalaron que ahora existen nuevas formulaciones más resistentes a los alimentos calientes, ya que muchos pacientes se quejaban de que con las comidas calientes se perdía el poder adhesivo de las cremas.

Los resultados correspondientes a las quejas de los pacientes, son mostrados en este trabajo únicamente a nivel informativo, ya que al no ir dirigida directamente la encuesta a los pacientes, no debemos estimar los resultados obtenidos con la misma fiabilidad estadística que en el resto de las variables. En todo caso, no dejan de aumentar la información requerida para que el odontólogo extrapole más de una consideración sobre el tema.

\section{Conclusiones}

En función de la revisión bibliográ- 
fica y las encuestas realizadas sobre una muestra de 40 farmacias, podemos exponer las siguientes conclusiones:

1. Según la bibliografía revisada, los adhesivos para dentaduras, son productos que pueden ayudar a los pacientes con prótesis removibles a conseguir mejorar su retención. Contribuyendo de esta manera, a mejorar su eficacia masticatoria, función fonatoria y autoestima.

2. No se le atribuye a los adhesivos ningún efecto relacionado con una mayor reabsorción ósea o daño sobre la mucosa

3. Se pueden recomendar los adhesivos a aquellos pacientes con enfermedades vesiculosas o aftas ya que contienen compuestos que logran disminuir el malestar. Distintos autores muestran que, el creci- miento de Candida albicans sólo se observa en pacientes que no contemplan una adecuada higiene de la prótesis.

4. En cuanto a la forma de presentación: el polvo es el más difícil de utilizar, las cremas son más cómodas y según algunos estudios logran mayor retención y las almohadillas logran sus mejores resultados cuando existe mayor incongruencia entre la prótesis y el reborde residual del paciente.

5. Según la información recogida en la encuesta realizada en las farmacias, la queja fundamental de los pacientes sigue siendo que los adhesivos no proporcionan la suficiente retención, sobre todo en el caso de las almohadillas.

6 En relación con la encuesta realizada, la crema es la forma de adhesivo más demandada por los pacientes, la que menos quejas recoge y con la que más satisfechos quedan, además de ser, en relación cantidad/precio, la forma de presentación más económica.

7. La venta de Adhesivos para prótesis completa comporta hoy en día, una parte importante de la actividad farmacéutica, apoyada de forma exhaustiva con campañas de televisión. Esta actividad resulta paradójica al relacionarla con la aparente reticencia a su prescripción por parte de los dentistas y, parece mantenerse ajena al ya establecimiento normalizado de las técnicas implantológicas.

8. El profesional debería, cuando así se requiera, tomar un papel más activo en la prescripción y orientación concreta del tipo de adhesivo a utilizar así como en cuanto a las instrucciones concretas a proporcionar al paciente.

\section{Bibliografia recomendada}

Para profundizar en la lectura de este tema, el/los autor/es considera/an interesantes los artículos que aparecen señalados del siguiente modo: *de interés **de especial interés.

1. Grasso J. Denture adhesive: changing attitudes. J Am Dent Assoc 1996;127:90-96.

2. Kennet AI. The use of denture adhesive as an aid to denture treatment. J Dent 1989;6:711-715.

3. Shay K. Denture Adhesive. J Am Dent Assoc 1991;122:70-76.

4. Ellis B, Al-Nakash S, Lamb DJ. The composition and rheology of denture adhesive. $J$ Dent 1980;2:109-118.

5. Swartz ML, Norman RD, Phillips RW. A method for measuring retention of denture adherents: An in vivo study. J Dent 1967;3:456-463.

6. Krishan K. A clinical evaluation of denture adhesive. J Dent 1967;6:550-558.

7. Ow RK, Bearn EM. A method studying the effect of adhesive on denture retention. J Dent 1983;3:332-337.

8. Chew CL. Retention of denture adhesive-an in vitro study. Oral Rehab 1990;17:425-434.

9. Grasso J, Rendell J, GAY T. Effect of denture adhesive on the retention and stability of maxillary dentures. J Dent 1994;72:399405 .
10. Tarbet W, Boone M, Schmidt N. Effect of a denture adhesive on complete denture dislodgement during mastication. J Dent 1990;4:374-378.

11. Coates AJ. Usage of denture adhesive. J Dent 2000,28:137-40.

12. Rendell J, Grasso J, Gay T. Retention and stability of the maxillary denture during function. J Dent 1995;4:344-347.

13. Neill DJ, Roberts BJ. The effect of denture fixatives on masticatory performance in complete denture patients. J Dent 1:219222.

14. Rendell J, Gay T, Grasso T, Baker R, Winston $J$ L. The effect of denture adhesive on mandibular movement during chewing. J Am Dent Assoc 2000;131:981-986.

15. Chew CL, Boone ME, SwartzML, Phillips RW. Denture adhesive: their effects on denture retention and stability. J Dent 1985;2:152-159.

16. Ghani P, Picton D. Some clinical investigations on retention forces of maxillary complete dentures with the use of denture fixative. Oral Rehab 1994;21:631-640.
17. Tarbet W, Sijvermna G, F Schmidt N. Maximum Incisal Biting Force in Denture Wearers as Influenced by Adequacy of Denture-bearing Tisues and the Use of an Adhesive. J Dent Res 1981;2:115-119.

18. Tarbet WJ, Grosman E. Observations of denture- supporting tissues during six months of denture adhesive wearing. $J$ Am Dent Assoc 1980;101: 789-791.

19. Berg E. A clinical comparison of four denture adhesive. Int J Prosthodont 1991; 4:449456.

20. Stafford GD. Efficiency of Denture Adhesive and Their Possible Influence on Oral Microorganisms. J Dental Res 1971;4: 832835.

21. Lo Muzio L, della Valle A, Mignogna MD, Pannone G, Bucci P, Bucci E, Sciubba J. The treatment of oral aphthous ulceration or erosive lichen planus with topical clobetasol propionate in three preparations: a clinical and pilot study on 54 patients. J Oral Pathol Med 2001;30:611-617.

22. Makihira S, Nikawa H, Satonobu SV, Jin CH, Hamada T. Crecimiento in vitro de la espe- 
cie Candida en adhesivos comerciales para dentaduras. Rev Int Prót Estomatol 2001,3; 2:178-182.

23. Nikawa H, Yanamoto T, Hamada T, Effect of components of resilient denture- lining materials on the growth, acid production and colonization of Candida albicans. Oral Rehab 1995;22: 817-824

24. Nikawa H, Yanamoto T, Hayashi S, Nikawa Y, Hamada T. Growth and/or acid production of Candida albicans on soft lining materials in vitro. Oral Rehab 1994;21:585-594.

25. Nikawa H, Hamada T, Yanamoto T, Kumagai
H. Effects of salivary or serum pellicles on the Candida albicans growth and biofilm formation on soft lining materials in vitro. J Oral Rehab 1997;24:594-604.

26. Nikawa H, Iwanaga I, Kameda M, Hamada T. In vitro evaluation of Candida albicans adherence to soft denture- lining materials. J Prost Dent 1992;68:6.

27. Nikawa H, Yanamoto T, Hamada T, Sadamoris, Aagrawal S. Cleansing efficacy of comercial denture cleansers: Ability to reduce Candida albicans biofilm activity. Int J Prosthodont 1995;8:524-527.
28. Uysal H, Altay OT, Alparslan N, Bilge A. Comparison of four different denture cushion adhesive- a subjetive study. J Oral Rehab 1998:25;204-208.

29. Kelsey CC, Lang BR, Wang RF. Examining patients' responses about the effectiveness of five denture adhesive pastes. J Am Dent Assoc 1997; 128:1532-1538.

30. Panagiotouni E, Pissiotis A, Kapari D, Kaloyannides A. Retentive ability of various denture adhesive materials: An in vitro study. The J Prost Dent. 1995;6: 578-585. 